\title{
Quality of life of female and male vegetarian and vegan endurance runners compared to omnivores - results from the NURMI study (step 2)
}

Patrick Boldt ${ }^{1}$, Beat Knechtle², Pantelis Nikolaidis ${ }^{3}$, Christoph Lechleitner ${ }^{4}$, Gerold Wirnitzer ${ }^{5}$, Claus Leitzmann ${ }^{6}$, Thomas Rosemann ${ }^{7}$ and Katharina Wirnitzer ${ }^{8,9^{*}}$ (D)

\begin{abstract}
Background: Health-related effects of a vegetarian or vegan diet are known to support parameters positively affecting exercise performance in athletes, whereas knowledge about psyche and wellbeing is sparse. Therefore, the aim of the Nutrition and Running High Mileage (NURMI) Study (Step 2) was to compare Quality of Life (QOL) scores among endurance runners following a vegetarian or vegan diet against those who adhere to an omnivorous diet.

Methods: The study was conducted following a cross-sectional design. A total of 281 recreational runners (159 women, 122 men) completed the WHOQOL-BREF questionnaire consisting of the domains physical health, psychological wellbeing, social relationships and environment, which generates scores on a scale from 4 to 20 . Data analysis was performed using ANOVA.

Results: It was found that 123 subjects followed an omnivorous diet and 158 adhered to a vegetarian/vegan diet. There were 173 runners who met the inclusion criteria ('NURMI-Runners'), among them 103 half-marathoners and 70 marathoners and ultramarathoners, as well as $10810 \mathrm{~km}$ runners as control group. Overall QOL scores were high $(\sim 16.62 \pm 1.91)$. Men had higher scores than women due to high scores in the physical health and psychological well-being dimensions. Adhering to an omnivorous diet affected environment scores for women and social relationships scores for men. A minor effect concerning race distance was observed in women, where half-marathoners had a higher environmental score than 10-km runners. A moderate dietxrace distance interaction on environment scores was shown for men.
\end{abstract}

Conclusions: The results revealed that endurance runners had a high QOL regardless of the race distance or diet choice. These findings support the notion that adhering to a vegetarian or vegan diet can be an appropriate and equal alternative to an omnivorous diet.

Trial registration: ISRCTN73074080. Registered 12th June 2015, retrospectively registered.

Keywords: Vegetarian, Vegan, Diet, Nutrition, Marathon running, Quality of life, Life satisfaction, WHOQOL-BREF

\footnotetext{
*Correspondence: katharina.wirnitzer@ph-tirol.ac.at; info@nurmi-study.com; katharina.wirnitzer@uibk.ac.at

${ }^{8}$ Centre for Research and Knowledge Management, Pedagogical University Tyrol, Feldstraße 1/ll, 6010 Innsbruck, Austria

${ }^{9}$ Department of Sport Science, University of Innsbruck, Innsbruck, Austria

Full list of author information is available at the end of the article
}

(c) The Author(s). 2018 Open Access This article is distributed under the terms of the Creative Commons Attribution 4.0 International License (http://creativecommons.org/licenses/by/4.0/), which permits unrestricted use, distribution, and reproduction in any medium, provided you give appropriate credit to the original author(s) and the source, provide a link to the Creative Commons license, and indicate if changes were made. The Creative Commons Public Domain Dedication waiver (http://creativecommons.org/publicdomain/zero/1.0/) applies to the data made available in this article, unless otherwise stated. 


\section{Background}

In the past 15 years, the number of participants in endurance running events, such as marathon or half-marathon races, has consistently been at a high level [1, 2]. More and more athletes among these adhere to a vegetarian or vegan diet $[3,4]$.

Health-related effects of a vegetarian or vegan diet have been found in athletes and are known to support parameters that positively affect exercise performance, such as physical fitness, resilience to chronic diseases, and weight control [5-7]. However, the knowledge about psychological and personal well-being is sparse. In order to generate an impression of an individual's life situation, meaning her/his needs, problems, concerns and emotional state, it can be appropriate to measure Quality of Life (QOL): a multidimensional concept that measures life satisfaction, including family, physical health, education, employment, wealth, religious beliefs, finance and environment factors $[8,9]$.

Variables that affect QOL include sex, dietary habits and physical activity [10-14]. The investigation of the impact of sex on QOL has yielded various results. Whereas in some studies higher QOL scores have been found in men [15-17], it has also been reported that women have higher scores [14]. The dimension of social relationships especially has had higher scores in female subjects [18]. In terms of sex differences in QOL-scores in endurance runners, no data has been made available yet.

The impact of diet choice on QOL has been investigated in the general population. A high QOL in general has been reported for both vegetarians [13] and vegans [10], as well as the beneficial effects of a meatless diet rich in fruits and vegetables on the QOL dimensions of 'depression' [19], 'anxiety' [20] and 'felt stress' [21, 22]. The rationale for this interconnectedness is that being a vegetarian or vegan is both a dietary habit and a lifestyle [23]. For many, vegetarianism and veganism are philosophies of how life should be lived and hence they are connected with certain characteristics, such as being health-conscious, liberal and having a humanistic view of the world [24]. As vegetarian and vegan dietary patterns are frequently considered in the dietary strategies of athletes [6], the purpose of the present study was to investigate to what extent findings from the general population apply to endurance athletes.

Similar results have been found for physical activity. It has been shown that physical activity in general can lead to a high QOL $[12,25]$. This has been confirmed by other studies investigating strength training [26], cycling [27] and musculoskeletal fitness [28]. As 'physical health' is an important requirement for life satisfaction, the synergistic effects of persistent adherence to a healthy diet and regular sport necessarily strongly influences QOL [23]. Further beneficial effects of an active lifestyle have been shown for numerous facets of QOL, such as 'life satisfaction' [29], 'sleep architecture' [30], 'felt stress' [31], 'anxiety' [32] and 'depression' [33].

All in all, some knowledge exists in terms of QOL and its interconnectedness with sex, diet choice and physical activity for the general population, suggesting there may be positive effects of a vegetarian and vegan diet on QOL. However, the data in terms of endurance runners and QOL is sparse. Therefore, in the Nutrition and Running High Mileage (NURMI) Study Step 2 we focused on the QOL of endurance runners, in particular in half-marathoners and marathoners. In the context of a rising number of athletes following a vegetarian or vegan diet $[3,4]$ and a lack of scientific literature concerning these groups, the aim of the study was to investigate QOL in endurance runners adhering to a vegetarian or vegan diet and compare them to endurance runners following a mixed diet.

Based on the findings from the general population, we hypothesized that QOL of omnivorous and vegetarian/ vegan endurance runners would be similar. Hence, a vegetarian or vegan diet could be an equivalent alternative to an omnivorous diet for endurance athletes.

\section{Methods}

\section{Experimental approach to the problem}

We assessed QOL using the WHOQOL-BREF [World Health Organization Quality of Life Assessment- brief (French: bref) version]. The WHOQOL-BREF is a shorter version of the original instrument that may be more convenient for use in large research studies or clinical trials [34]. The WHOQOL-BREF's validity is well established and has been confirmed by a number of studies $[9,35,36]$.

The WHOQOL-BREF instrument comprises 26 items, which measure the following broad domains: physical health (i.e. activities of daily living, dependence on medicinal substances and medical aids, energy and fatigue, mobility, pain and discomfort, sleep and rest, work capacity; DOM 1), psychological well-being (i.e. bodily image and appearance, negative feelings, positive feelings, self-esteem, spirituality/religion/personal beliefs, thinking, learning, memory and concentration; DOM 2), social relationships (i.e. personal relationships, social support, sexual activity; DOM 3) and environment (i.e. financial resources, freedom, physical safety and security, health and social care: accessibility and quality, home environment, opportunities for acquiring new information and skills, participation in and opportunities for recreation/leisure activities, physical environment (i. e. pollution/noise/traffic/climate, transport; DOM 4).

Each item was rated on a 5-point Likert scale. The typical Likert scale is a 5-point ordinal scale used by respondents to rate the degree to which they agree or 
disagree with a statement (i.e. higher scores denote stronger agreement or disagreement, respectively).

Afterwards, four domain scores were derived. Raw domain scores for the WHOQOL were transformed to a 4-20 score and scaled in a positive direction (i.e. higher scores denote higher QOL). The mean score of items within each domain was used to calculate the domain score [34].

\section{Subjects}

The NURMI Study was conducted in three steps following a cross-sectional design. We recruited endurance runners mainly from German-speaking countries, such as Germany, Austria and Switzerland. In addition, we approached people from all over Europe. The subjects were contacted mainly via social media, websites of the organizers of marathon events, online running communities, email-lists, runners' magazines as well as magazines for health, vegetarian and/or vegan nutrition and lifestyle, sports fairs, fairs on vegetarian and vegan nutrition and lifestyle, and through personal contacts.

The study protocol [4] was approved by the ethics board of St. Gallen, Switzerland on May 6, 2015 (EKSG 14/145). The trial registration number is ISRCTN73074080.

\section{Procedures}

The participants completed an online survey within the NURMI Study Step 2, provided in German and English, which was available on https://www.nurmi-study.com/en from February 1st 2015 until December 31st 2015.

The survey started with a written description of the procedure and participants gave their informed consent to take part in the study. Afterwards, they completed the WHOQOL-BREF questionnaire (for further information see below) including questions concerning physical health, psychological well-being, social relationships and environment. In addition, we asked for age, sex and preferred diet.

For successful participation, the following criteria were required: written informed consent (1), at least 18 years of age (2), WHOQOL-BREF questionnaire completed (3), successful participation in a running event of either half-marathon or marathon distance in the past two years (4). Incomplete and inconsistent data sets were eliminated. Those who met all inclusion criteria but named a $10-\mathrm{km}$ race as their running event were kept as controls. In the following they are called ' $10-\mathrm{km}$ control group', whereas those who met the inclusion criteria to the full extent are referred to as 'NURMI-Runners'.

Participants were classified into two diet groups: omnivorous diet (commonly known as Western diet, no dietary restrictions) versus vegetarian (no meat)/vegan (no products from animal sources, such as meat, fish, milk and dairy products, eggs and honey) diet [5]. Moreover, they were categorized into three race distances: $10 \mathrm{~km}$, half-marathon and marathon/ultramarathon.

\section{Statistical analyses}

The statistical software IBM SPSS version 23.0 (SPSS, Chicago, USA) and GraphPad Prism version 7.0 (GraphPad Software, San Diego, USA) performed all statistical analyses. The Kolmogorov-Smirnoff test of normality and visual inspection of normal Q-Q plots examined the normality of all variables. Mean values and standard deviation (SD) were calculated for all variables. The student $\mathrm{t}$-test examined sex differences in the four domains of WHOQOL and Cohen's $d(d \leq 0.2$, trivial; $0.2<d \leq 0.6$, small; $0.6<d \leq 1.2$, moderate; $1.2<d \leq 2.0$, large; and $d>2.0$, very large) evaluated the magnitude of these differences. A two-way ANOVA, followed by a Bonferroni post-hoc analysis, examined the main effects of nutrition and race distance, the nutrition*race distance interaction on WHOQOL. The magnitude of differences in the ANOVA was evaluated using eta squared $\left(\eta^{2}\right)$ as trivial $\left(\eta^{2}<0.01\right)$, small $\left(0.01 \leq \eta^{2}<0.06\right)$, moderate $\left(0.06 \leq \eta^{2}<0.14\right)$ and large $\left(\eta^{2} \geq 0.14\right)$. The level of statistical significance was set at $p \leq 0.05$.

\section{Results}

A total of 317 endurance runners completed the survey, of whom 281 (159 women and 122 men) with a mean age of $40 \pm 11$ years remained after data clearance. Their countries of origin were Germany $(n=200)$, Switzerland $(n=14)$, Austria $(n=50)$ and some others $(n=17$; Belgium, Brazil, Canada, Italy, Luxemburg, Netherlands, Poland, Spain, United Kingdom).

With regard to dietary subgroups, 123 subjects followed an omnivorous diet and 158 adhered to a vegetarian/vegan diet. Concerning race distances, there were 173 NURMI-Runners (103 half-marathoners, 70 marathoners/ultramarathoners) and 108 members of the $10-\mathrm{km}$ control group. Characteristics of our subjects are presented in Table 1.

\section{Sex differences in quality of life}

Scores for physical health were $17.6 \pm 1.4$ (85.13\%) in women and $18.0 \pm 1.3(87.24 \%)$ in men, for psychological wellbeing $16.0 \pm 2.1(74.71 \%)$ and $16.8 \pm 1.8$ (80.16\%), for social relationships $15.5 \pm 2.6(71.59 \%)$ and $15.4 \pm 2.9$ (70.97\%), and for environment $16.8 \pm 1.6$ (80.05\%) and $17.0 \pm 1.7(80.99 \%)$. Men had higher scores in physical health $(p=0.037, \mathrm{~d}=0.26)$ and psychological wellbeing $(p<0.001, \mathrm{~d}=0.45)$, but there were no differences with regard to social relationships counts $(p=0.761, \mathrm{~d}=0.03)$ and environment scores $(p=0.445, \mathrm{~d}=0.09)$ compared to women (Fig. 1a, b, 2a, b). 
Table 1 Anthropometric and Demographic Characteristics of the Subjects Displayed by Diet Group

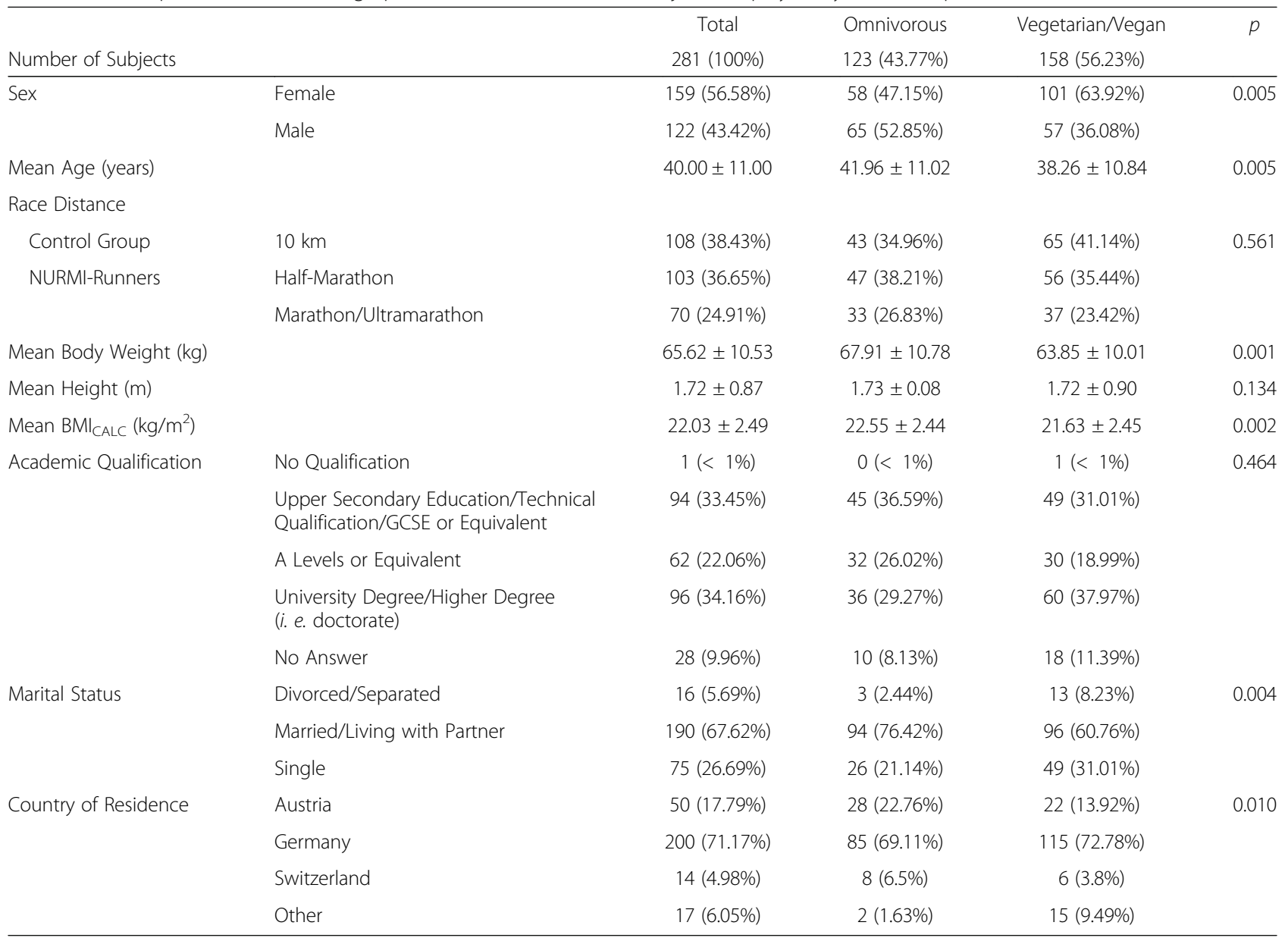

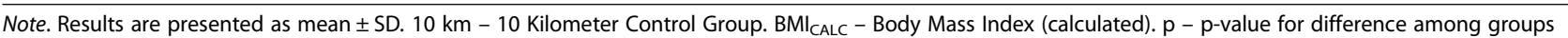

\section{Main effects of diet choice on quality of life}

Scores for physical health were $17.5 \pm 1.5(84.6 \%)$ in female omnivorous runners, $18.0 \pm 1.4(87.4 \%)$ in male omnivorous runners, $17.7 \pm 1.5(85.4 \%)$ in female vegetarian/vegan runners and $17.9 \pm 1.1(87.0 \%)$ in male vegetarian/vegan runners. With regard to psychological wellbeing, mean scores were $16.4 \pm 2.0(77.3 \%)$ in female omnivorous runners, $17.0 \pm 1.9(81.5 \%)$ in male omnivorous runners, $15.7 \pm 2.0$ (73.3\%) in female vegetarian/ vegan runners and $16.6 \pm 1.7(78.6 \%)$ in male vegetarian/ vegan runners. Social relationships scores were $15.5 \pm 2.3$ (71.7\%) in female omnivorous runners, $15.9 \pm 2.7$ (74.4\%) in male omnivorous runners, $15.5 \pm 2.8(71.6 \%)$ in female vegetarian/vegan runners and $14.7 \pm 2.9(67.1 \%)$ in male vegetarian/vegan runners. In terms of environment, mean scores were $17.2 \pm 1.6(82.2 \%)$ in female omnivorous runners, $17.2 \pm 1.8(82.3 \%)$ in male omnivorous runners, $16.6 \pm 1.6(78.8 \%)$ in female vegetarian/vegan runners and $16.7 \pm 1.6(79.6 \%)$ in male vegetarian/vegan runners (Fig. 1a and b).

No major effect of diet on physical health $(p=0.248$, $\eta^{2}=0.009$ and $\left.p=0.844, \eta^{2}<0.001\right)$, and psychological wellbeing ( $p=0.164, \eta^{2}=0.013$ and $\left.p=0.246, \eta^{2}=0.012\right)$ in both sexes, on social relationships in women $(p=0.691$, $\left.\eta^{2}=0.001\right)$ or on environment in men $(p=0.358$, $\left.\eta^{2}=0.007\right)$ was observed. However, a minor effect of diet on social relationships in men $\left(p=0.047, \eta^{2}=0.034\right)$ and environment in women $\left(p=0.043, \eta^{2}=0.027\right)$ was shown with a higher score in the omnivorous diet (Fig. 1a, Fig. 1b). Mean scores for each item are shown in Table 2.

\section{Main effects of race distance on quality of life and dietxrace distance interaction}

Mean scores in physical health were $17.5 \pm 1.5$ (84.3\%) in female members of the 10-km control group, $18.0 \pm 1.1$ (87.4\%) in male members of the $10-\mathrm{km}$ control group, $17.7 \pm 1.3(85.6 \%)$ in female half marathoners, $18.1 \pm 1.2$ $(87.8 \%)$ in male half marathoners, $17.8 \pm 1.3(86.5 \%)$ in female marathoners/ultramarathoners and $17.8 \pm 1.6$ $(86.4 \%)$ in male marathoners/ultramarathoners. In terms of psychological wellbeing, mean scores were $15.8 \pm 2.0$ $(73.9 \%)$ in female members of the $10-\mathrm{km}$ control group, $16.7 \pm 2.0(79.1 \%)$ in male members of the $10-\mathrm{km}$ control 

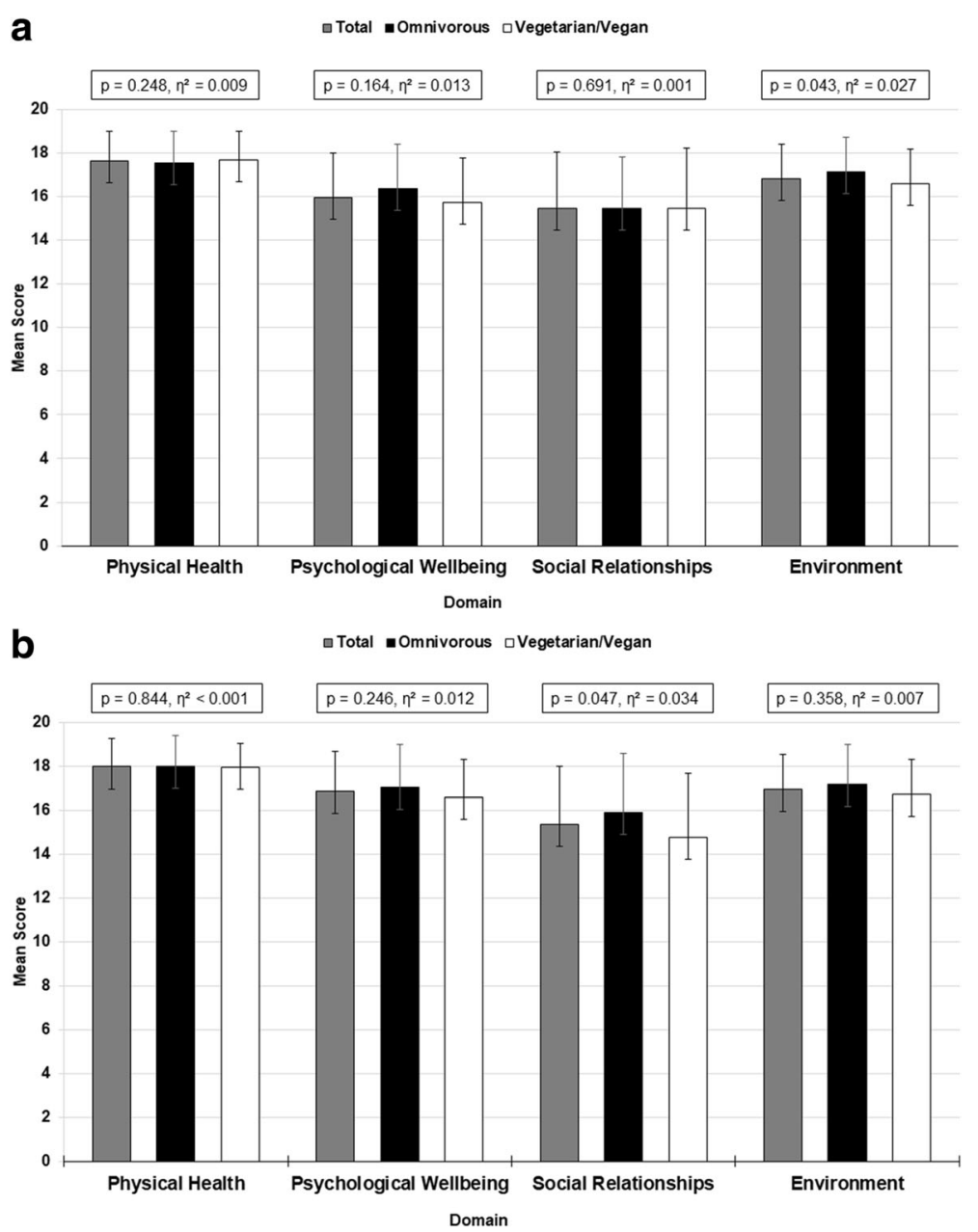

Fig. 1 a Mean WHOQOL-BREF-Domain Scores of Women Displayed by Diet Group. Note. Results are presented as mean \pm SD. $p-p$-value for differences between groups. $\eta^{2}$ - effect size. b. Mean WHOQOL-BREF-Domain Scores of Men Displayed by Diet Group. Note. Results are presented as mean $\pm S D$. $p-p$-value for differences between groups. $\eta^{2}$ - effect size

group, $15.8 \pm 2.1(74.0 \%)$ in female half marathoners, $16.9 \pm 2.0(80.7 \%)$ in male half marathoners, $16.5 \pm 2.1$ $(78.4 \%)$ in female marathoners/ultramarathoners and $16.9 \pm 1.6(80.4 \%)$ in male marathoners/ultramarathoners. Mean scores in social relationships were $15.4 \pm 2.6$ (71.3\%) in female members of the 10- $\mathrm{km}$ control group, $15.0 \pm 2.6$ (68.7\%) in male members of the $10-\mathrm{km}$ control group, $15.4 \pm 2.8(71.5 \%)$ in female half marathoners, $15.6 \pm 3.0$ $(72.4 \%)$ in male half marathoners, $15.6 \pm 2.5(72.5 \%)$ in female marathoners/ultramarathoners and $15.4 \pm 1.7$ (71.1\%) in male marathoners/ultramarathoners. With regard to environment, mean scores were $16.4 \pm 1.6$ (77.6\%) in female members of the 10-km control group, 16.4 \pm 1.8 (77.4\%) in male members of the $10-\mathrm{km}$ control group, $16.4 \pm 1.5(77.6 \%)$ in female half marathoners, $17.28 \pm 1.7$ $(83.0 \%)$ in male half marathoners, $17.2 \pm 1.3(82.3 \%)$ in female marathoners/ultramarathoners and $17.1 \pm 1.7$ $(81.6 \%)$ in male marathoners/ultramarathoners.
No major effect of race distance on physical health $\left(p=0.586, \eta^{2}=0.007\right.$ and $\left.p=0.847, \eta^{2}=0.003\right)$, psychological wellbeing ( $p=0.379, \eta^{2}=0.013$ and $\left.p=0.818, \eta^{2}=0.003\right)$, or social relationships $\left(p=0.986, \eta^{2}<0.001\right.$ and $p=0.838$, $\eta^{2}=0.003$ ) for women and men, respectively was shown.

Also, no effect of race distance on environment for men was found $\left(p=0.121, \eta^{2}=0.036\right)$. However, a minor effect was observed for women $\left(p=0.014, \eta^{2}=0.054\right)$, where half-marathoners had a higher environment score than the members of the 10-km control group (Fig. 2a, $2 b$, Table 3).

No diet $\times$ race distance interaction on physical health $(p=0.346, \eta 2=0.014$ and $p=0.060, \eta 2=0.047)$, psychological well-being $(p=0.672, \eta 2=0.005$ and $p=0.026$, $\eta 2=0.061)$ or social relationships $(p=0.490, \eta 2=0.009$ and $p=0.112, \eta 2=0.037$ ) for women or men, respectively, was observed. A moderate diet $\times$ race distance interaction on environment score was shown for men $(p=$ 
Table 2 Mean Likert-Scores of the WHOQOLBREF-Items Displayed by Diet Group

\begin{tabular}{|c|c|c|c|c|c|}
\hline Question & Total & Omnivorous & Vegetarian/Negan & $p$ & $\eta^{2}$ \\
\hline \multicolumn{6}{|c|}{ How would you rate your Quality of Life? ${ }^{1}$} \\
\hline Women & $4.33 \pm 0.58$ & $4.38 \pm 0.59$ & $4.30 \pm 0.58$ & 0.450 & 0.00 \\
\hline Men & $4.48 \pm 0.65$ & $4.46 \pm 0.74$ & $4.49 \pm 0.53$ & & \\
\hline \multicolumn{6}{|c|}{ How satisfied are you with your health? ${ }^{2}$} \\
\hline Women & $4.09 \pm 0.93$ & $4.03 \pm 1.11$ & $4.13 \pm 0.82$ & 0.176 & 0.00 \\
\hline Men & $4.30 \pm 0.95$ & $4.11 \pm 1.13$ & $4.51 \pm 0.68$ & & \\
\hline
\end{tabular}

To what extent do you feel that physical pain prevents you from doing what you need to do? ${ }^{3}$

$\begin{array}{llllll}\text { Women } & 1.33 \pm 0.58 & 1.31 \pm 0.65 & 1.35 \pm 0.54 & 0.145 & 0.008 \\ \text { Men } & 1.34 \pm 0.57 & 1.42 \pm 0.62 & 1.25 \pm 0.52 & \end{array}$

How much do you need any medical treatment to function in your daily life? ${ }^{3}$

$\begin{array}{lllll}\text { Women } & 1.15 \pm 0.42 & 1.24 \pm 0.55 & 1.10 \pm 0.34 & 0.357 \\ \text { Men } & 1.22 \pm 0.67 & 1.23 \pm 0.68 & 1.21 \pm 0.65 & 0.003 \\ \text { How much do you enjoy life? }^{3} & & & & \\ \quad \text { Women } & 4.17 \pm 0.71 & 4.31 \pm 0.79 & 4.09 \pm 0.67 & 0.354 \\ \text { Men } & 4.17 \pm 0.71 & 4.20 \pm 0.73 & 4.14 \pm 0.69 & 0.003\end{array}$

To what extent do you feel your life to be meaningful? ${ }^{3}$

$\begin{array}{llllll}\text { Women } & 4.16 \pm 0.65 & 4.19 \pm 0.71 & 4.15 \pm 0.62 & 0.487 & 0.002 \\ \text { Men } & 4.28 \pm 0.77 & 4.31 \pm 0.86 & 4,25 \pm 0.67 & \end{array}$

How well are you able to concentrate? ${ }^{3}$

$\begin{array}{lllll}\text { Women } & 3.89 \pm 0.75 & 4.02 \pm 0.82 & 3.81 \pm 0.72 & 0.217 \\ \text { Men } & 4.01 \pm 0.72 & 4.00 \pm 0.78 & 4.02 \pm 0.65 & 0.006\end{array}$

How safe do you feel in your daily life? ${ }^{3}$

$\begin{array}{lllll}\text { Women } & 4.16 \pm 0.65 & 4.19 \pm 0.71 & 4.15 \pm 0.62 & 0.904 \\ \text { Men } & 4.28 \pm 0.77 & 4.31 \pm 0.86 & 4.25 \pm 0.67 & <.001\end{array}$

How healthy is your physical environment? ${ }^{3}$

$\begin{array}{lllll}\text { Women } & 3.91 \pm 0.78 & 3.93 \pm 0.75 & 3.90 \pm 0.81 & 0.190 \\ \text { Men } & 3.98 \pm 0.80 & 4.11 \pm 0.80 & 3.82 \pm 0.81 & 0.006\end{array}$

Do you have enough energy for everyday life? ${ }^{4}$

Women

$$
4.18 \pm 0.64
$$

$4.16 \pm 0.70$

$4.19 \pm 0.61$

0.893

$4.35 \pm 0.61$

Are you able to accept your bodily appearance? ${ }^{4}$

Women

Have you enough money to meet your needs? ${ }^{4}$

Women
Men

$$
\begin{aligned}
& 4.00 \pm 0.70 \\
& 4.24 \pm 0.69
\end{aligned}
$$$$
3.79 \pm 0.73
$$$$
4.02 \pm 0.69
$$$$
4.28 \pm 0.66
$$$$
4.19 \pm 0.72
$$

$4.14 \pm 0.63$

$3.94 \pm 0.86$

$3.89 \pm 0.88$

$3.91 \pm 0.77$

How available to you is the information that you need in your day-to-day life? ${ }^{4}$

Women

$$
\begin{aligned}
& 4.65 \pm 0.50 \\
& 4.71 \pm 0.46
\end{aligned}
$$

$4.79 \pm 0.43$

$4.72 \pm 0.46$

$4.57 \pm 0.54$

0.093

0.010

To what extent do you have the opportunity for leisure activities? ${ }^{4}$

Women

$4.33 \pm 0.70$
$4.28 \pm 0.69$

$4.43 \pm 0.73$

$4.22 \pm 0.75$

$4.78 \pm 0.46$
Women
$4.84 \pm 0.39$
$4.70 \pm 0.46$

$4.28 \pm 0.68$

0.089

0.010

$4.35 \pm 0.61$

$4.87 \pm 0.34$

0.198

0.006 
Table 2 Mean Likert-Scores of the WHOQOLBREF-Items Displayed by Diet Group (Continued)

\begin{tabular}{|c|c|c|c|c|c|}
\hline Question & Total & Omnivorous & Vegetarian/Negan & p & $\eta^{2}$ \\
\hline Men & $4.88 \pm 0.33$ & $4.89 \pm 0.32$ & $4.86 \pm 0.35$ & & \\
\hline \multicolumn{6}{|c|}{ How satisfied are you with your sleep? ${ }^{2}$} \\
\hline Women & $3.82 \pm 0.91$ & $3.64 \pm 0.85$ & $3.92 \pm 0.95$ & \multirow[t]{2}{*}{0.030} & \multirow[t]{2}{*}{0.017} \\
\hline Men & $3.96 \pm 0.85$ & $4.05 \pm 0.85$ & $3.86 \pm 0.86$ & & \\
\hline \multicolumn{6}{|c|}{ How satisfied are you with your ability to perform your daily living activities? ${ }^{2}$} \\
\hline Women & $4.26 \pm 0.72$ & $4.36 \pm 0.62$ & $4.20 \pm 0.78$ & \multirow[t]{2}{*}{0.197} & \multirow[t]{2}{*}{0.006} \\
\hline Men & $4.34 \pm 0.58$ & $4.32 \pm 0.62$ & $4.37 \pm 0.53$ & & \\
\hline \multicolumn{6}{|c|}{ How satisfied are you with your capacity for work? ${ }^{2}$} \\
\hline Women & $4.23 \pm 0.71$ & $4.28 \pm 0.67$ & $4.23 \pm 0.71$ & \multirow[t]{2}{*}{0.717} & \multirow[t]{2}{*}{$<0.001$} \\
\hline Men & $4.46 \pm 0.65$ & $4.52 \pm 0.68$ & $4.39 \pm 0.61$ & & \\
\hline \multicolumn{6}{|c|}{ How satisfied are you with yourself? ${ }^{2}$} \\
\hline Women & $3.75 \pm 0.60$ & $4.00 \pm 0.62$ & $3.94 \pm 0.82$ & \multirow[t]{2}{*}{0.821} & \multirow[t]{2}{*}{$<0.001$} \\
\hline Men & $4.14 \pm 0.72$ & $4.18 \pm 0.79$ & $4.09 \pm 0.64$ & & \\
\hline \multicolumn{6}{|c|}{ How satisfied are you with your personal relationships? ${ }^{2}$} \\
\hline Women & $4.02 \pm 0.82$ & $4.09 \pm 0.76$ & $3.98 \pm 0.86$ & \multirow[t]{2}{*}{0.366} & \multirow[t]{2}{*}{0.003} \\
\hline Men & $4.08 \pm 0.80$ & $4.22 \pm 0.79$ & $3.93 \pm 0.83$ & & \\
\hline \multicolumn{6}{|c|}{ How satisfied are you with your sex life? ${ }^{2}$} \\
\hline Women & $3.58 \pm 0.96$ & $3.52 \pm 0.98$ & $3.62 \pm 0.95$ & \multirow[t]{2}{*}{0.170} & \multirow[t]{2}{*}{0.007} \\
\hline Men & $3.58 \pm 1.09$ & $3.69 \pm 1.11$ & $3.46 \pm 1.08$ & & \\
\hline \multicolumn{6}{|c|}{ How satisfied are you with the support you get from your friends? ${ }^{2}$} \\
\hline Women & $3.99 \pm 0.73$ & $4.00 \pm 0.62$ & $3.98 \pm 0.79$ & \multirow[t]{2}{*}{0.067} & \multirow[t]{2}{*}{0.012} \\
\hline Men & $3.85 \pm 0.76$ & $4.02 \pm 0.72$ & $3.67 \pm 0.81$ & & \\
\hline \multicolumn{6}{|c|}{ How satisfied are you with the conditions of your living place? ${ }^{2}$} \\
\hline Women & $4.13 \pm 0.90$ & $4.28 \pm 0.89$ & $4.04 \pm 0.91$ & \multirow[t]{2}{*}{0.386} & \multirow[t]{2}{*}{0.003} \\
\hline Men & $4.14 \pm 0.91$ & $4.34 \pm 0.95$ & $3.91 \pm 0.88$ & & \\
\hline \multicolumn{6}{|c|}{ How satisfied are you with your access to health services? ${ }^{2}$} \\
\hline Women & $4.19 \pm 0.81$ & $4.22 \pm 0.75$ & $4.18 \pm 0.84$ & \multirow[t]{2}{*}{0.654} & \multirow[t]{2}{*}{0.001} \\
\hline Men & $4.25 \pm 0.75$ & $4.31 \pm 0.84$ & $4.18 \pm 0.66$ & & \\
\hline \multicolumn{6}{|c|}{ How satisfied are you with your transport? ${ }^{2}$} \\
\hline Women & $4.25 \pm 0.79$ & $4.31 \pm 0.63$ & $4.22 \pm 0.87$ & \multirow[t]{2}{*}{0.830} & $<0.001$ \\
\hline Men & $4.36 \pm 0.78$ & $4.38 \pm 0.83$ & $4.33 \pm 0.72$ & & \\
\hline How often o & blue mood, $d$ & xiety, depressi & & & \\
\hline Women & $2.23 \pm 0.83$ & $1.95 \pm 0.76$ & $2.23 \pm 0.83$ & 0.261 & 0.005 \\
\hline Men & $1.63 \pm 0.88$ & $1.54 \pm 0.94$ & $1.74 \pm 0.81$ & & \\
\hline
\end{tabular}

Note. Results are presented as mean \pm SD. $p-p$-value for ANOVA test. $\eta^{2}$ - effect size

${ }^{1} 1=$ very poor, $2=$ poor, $3=$ neither poor nor good, $4=$ good, $5=$ very good

${ }^{2} 1=$ very dissatisfied, 2 = dissatisfied, $3=$ neither satisfied nor dissatisfied, $4=$ satisfied, $5=$ very satisfied

${ }^{3} 1=$ not at all, $2=\mathrm{a}$ little, $3=\mathrm{a}$ moderate amount, $4=$ very much, $5=$ an extreme amount

${ }^{4} 1=$ not at all, $2=$ a little, $3=$ moderately, $4=$ mostly, $5=$ completely

${ }^{5} 1=$ never, 2 = seldom, 3 = quite often, $4=$ very often, $5=$ always

0.013, $\eta 2=0.072)$, but no interaction was found for women $(p=0.925, \eta 2=0.001)$.

\section{Discussion}

This study aimed to investigate QOL of female and male endurance runners following a vegetarian or vegan diet and to compare it to female and male endurance runners adhering to an omnivorous diet. The hypothesis was that QOL would be equal in both groups and hence a vegetarian or vegan diet could be an equivalent alternative to an omnivorous diet.

The main findings were that $(i)$ men had higher scores in physical health and psychological well-being as compared to women, but there were no sex differences in 

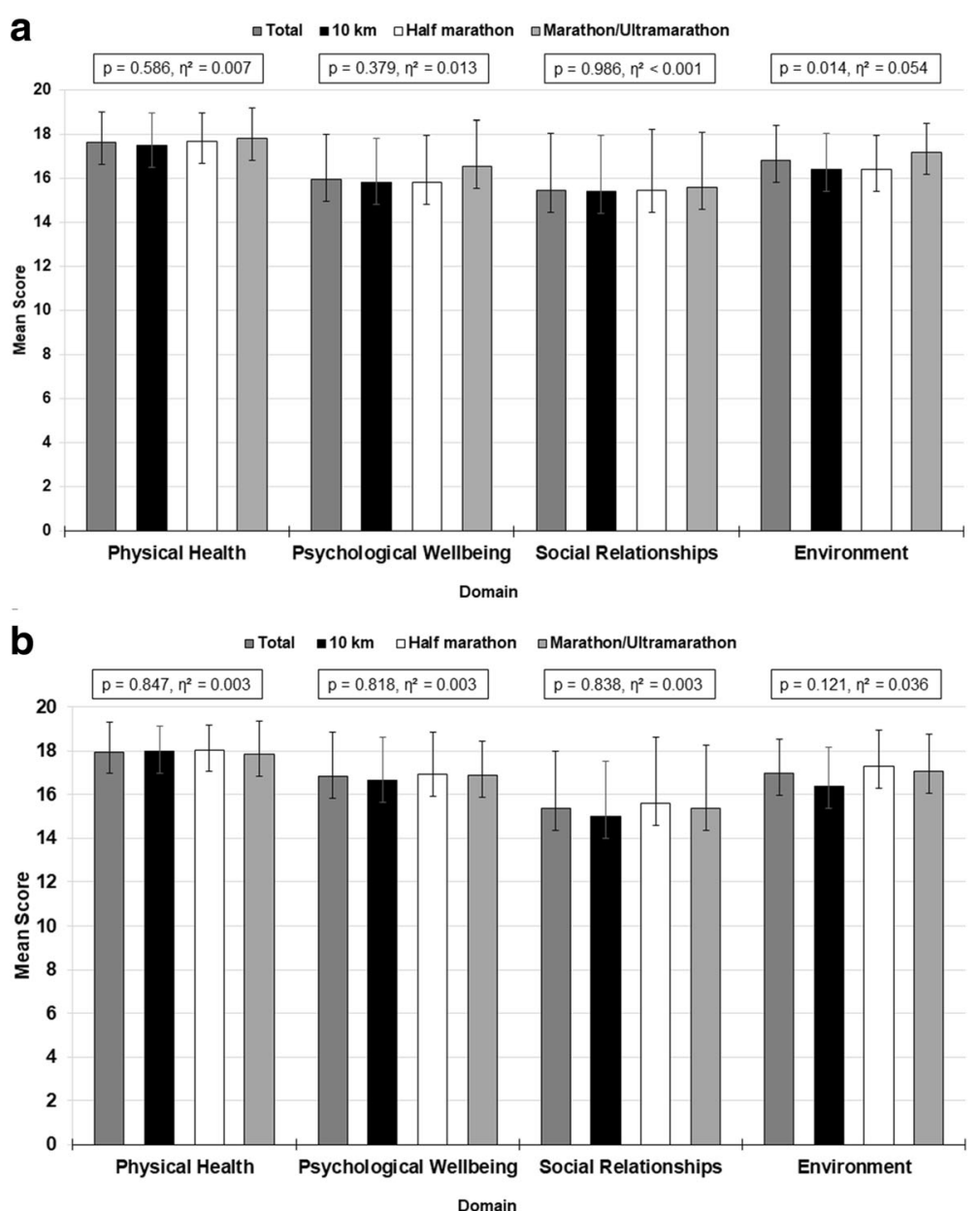

Fig. 2 a Mean WHOQOL-BREF-Domain Scores of Women Displayed by Race Distance. Note. Results are presented as mean \pm SD. $p-p$-value for differences between groups. $\eta^{2}$ - effect size. $\mathbf{b}$ Mean WHOQOL-BREF-Domain Scores of Men Displayed by Race Distance. Note. Results are presented as mean \pm SD. $p-p$-value for differences between groups. $\eta^{2}$ - effect size

terms of social relationships counts and environment scores, (ii) no major effect of diet on physical health and psychological wellbeing in either sex, on social relationships for women or on environment for men, was observed, (iii) a minor effect of diet on social relationships for men and environment for women was shown, with higher score for omnivores, (iv) no major effect of race distance on physical health, psychological and social relationships was shown for either women or men, (v) no effect of race distance on environment for men was found, but a minor effect was observed for women, where half-marathoners had a higher environment score than the members of the $10-\mathrm{km}$ control group, (vi) no diet $\times$ race distance interaction on physical health, psychological wellbeing or social relationships was observed for women or men, and (vii) a moderate diet $\times$ race distance interaction on environment score was shown for men, although no interaction was found in women.

\section{Sex differences in quality of life}

A first important finding was that male endurance runners have higher overall QOL scores compared to female endurance runners, mainly based on higher counts in the domains of physical health and psychological wellbeing. These sex differences have been observed in other studies as well $[11,16,17]$, particularly relating to psychological factors [37].

A potential explanation could be that women are more emotional and sensitive to perceived pressure, as compared to men $[38,39]$. It has been shown that women are more willing to report symptoms [40] whereas men often stick to traditional role concepts. They think society expects them to be strong and self-reliant ('Macho-Concept', 'Social desirability'), but they must not complain about symptoms or other 'sissy-stuff' $[41,42]$. The phenomenon that women report poorer (physical) health is well known and is termed 'gender paradox'. Although women live 
Table 3 Mean Likert-Scores of the WHQOLBREF-Items Displayed by Race Distance

\begin{tabular}{|c|c|c|c|c|c|c|}
\hline \multirow{2}{*}{ Question } & \multirow[b]{2}{*}{ Total } & \multirow{2}{*}{$\frac{\text { Control Group }}{10 \mathrm{KM}}$} & \multicolumn{4}{|c|}{ NURMI-Runners } \\
\hline & & & $\mathrm{HM}$ & M/UM & $p$ & $\eta^{2}$ \\
\hline \multicolumn{7}{|c|}{ How would you rate your Quality of Life? ${ }^{1}$} \\
\hline Women & $4.33 \pm 0.58$ & $4.33 \pm 0.58$ & $4.26 \pm 0.62$ & $4.44 \pm 0.51$ & 0.111 & 0.016 \\
\hline Men & $4.48 \pm 0.65$ & $4.45 \pm 0.58$ & $4.59 \pm 0.56$ & $4.37 \pm 0.79$ & & \\
\hline \multicolumn{7}{|c|}{ How satisfied are you with your health? ${ }^{2}$} \\
\hline Women & $4.09 \pm 0.93$ & $3.93 \pm 1.03$ & $4.18 \pm 0.89$ & $4.37 \pm 0.69$ & 0.111 & 0.016 \\
\hline Men & $4.30 \pm 0.95$ & $4.39 \pm 0.68$ & $4.30 \pm 1.03$ & $4.21 \pm 1.04$ & & \\
\hline
\end{tabular}

To what extent do you feel that physical pain prevents you from doing what you need to do? ${ }^{3}$

\begin{tabular}{|c|c|c|c|c|c|}
\hline Women & $4.67 \pm 0.58$ & $4.67 \pm 0.58$ & $4.72 \pm 0.53$ & $4.56 \pm 0.70$ & 0.314 \\
\hline Men & $4.66 \pm 0.57$ & $4.85 \pm 0.36$ & $4.67 \pm 0.56$ & $4.51 \pm 0.69$ & \\
\hline
\end{tabular}

How much do you need any medical treatment to function in your daily life? ${ }^{3}$

\begin{tabular}{|c|c|c|c|c|c|}
\hline Women & $4.82 \pm 0.43$ & $4.83 \pm 0.42$ & $4.89 \pm 0.45$ & $4.81 \pm 0.40$ & 0.746 \\
\hline Men & $4.78 \pm 0.66$ & $4.82 \pm 0.47$ & $4.85 \pm 0.63$ & $4.67 \pm 0.81$ & \\
\hline
\end{tabular}

How much do you enjoy life? ${ }^{3}$

\begin{tabular}{|c|c|c|c|c|c|}
\hline Women & $4.17 \pm 0.71$ & $4.13 \pm 0.72$ & $4.18 \pm 0.73$ & $4.26 \pm 0.66$ & 0.924 \\
\hline Men & $4.17 \pm 0.71$ & $4.12 \pm 0.60$ & $4.11 \pm 0.90$ & $4.28 \pm 0.55$ & \\
\hline
\end{tabular}

To what extent do you feel your life to be meaningful? ${ }^{3}$

$\begin{array}{llllll}\text { Women } & 4.11 \pm 0.78 & 4.05 \pm 0.84 & 4.12 \pm 0.76 & 4.26 \pm 0.66 & 0.950 \\ \text { Men } & 4.31 \pm 0.81 & 4.24 \pm 0.71 & 4.30 \pm 1.03 & 4.37 \pm 0.62 & <.001\end{array}$

How well are you able to concentrate? ${ }^{4}$

$\begin{array}{lllllll}\text { Women } & 3.89 \pm 0.76 & 3.87 \pm 0.74 & 3.89 \pm 0.80 & 3.93 \pm 0.73 & 0.903 & 0.001 \\ \text { Men } & 4.01 \pm 0.71 & 3.97 \pm 0.59 & 3.96 \pm 0.70 & 4.09 \pm 0.81 & \end{array}$

How safe do you feel in your daily life? ${ }^{4}$

$\begin{array}{llllll}\text { Women } & 4.16 \pm 0.66 & 4.04 \pm 0.71 & 4.23 \pm 0.60 & 4.37 \pm 0.57 & 0.267 \\ \text { Men } & 4.28 \pm 0.76 & 4.30 \pm 0.68 & 4.26 \pm 0.91 & 4.28 \pm 0.67 & 0.010\end{array}$

How healthy is your physical environment? ${ }^{4}$

$\begin{array}{llllll}\text { Women } & 3.91 \pm 0.78 & 3.75 \pm 0.84 & 4.07 \pm 0.73 & 4.04 \pm 0.65 & 0.220 \\ \text { Men } & 3.98 \pm 0.80 & 4.00 \pm 0.71 & 4.07 \pm 0.88 & 3.86 \pm 0.77 & 0.011\end{array}$

Do you have enough energy for everyday life? ${ }^{4}$

\begin{tabular}{|c|c|c|c|c|c|}
\hline Women & $4.18 \pm 0.64$ & $4.07 \pm 0.70$ & $4.28 \pm 0.56$ & $4.26 \pm 0.59$ & 0.888 \\
\hline Men & $4.34 \pm 0.59$ & $4.18 \pm 0.64$ & $4.37 \pm 0.53$ & $4.44 \pm 0.59$ & \\
\hline
\end{tabular}

Are you able to accept your bodily appearance? ${ }^{4}$

\begin{tabular}{|c|c|c|c|c|c|c|}
\hline Women & $4.00 \pm 0.70$ & $3.95 \pm 0.70$ & $4.00 \pm 0.71$ & $4.15 \pm 0.72$ & \multirow[t]{2}{*}{0.349} & \multirow[t]{2}{*}{0.008} \\
\hline Men & $4.24 \pm 0.64$ & $4.15 \pm 0.80$ & $4.35 \pm 0.53$ & $4.19 \pm 0.63$ & & \\
\hline \multicolumn{7}{|c|}{ lave you enough money to meet your needs? ${ }^{4}$} \\
\hline Women & $3.98 \pm 0.79$ & $3.91 \pm 0.87$ & $4.00 \pm 0.73$ & $4.15 \pm 0.66$ & \multirow[t]{2}{*}{0.826} & 0.001 \\
\hline Men & $3.93 \pm 0.82$ & $3.79 \pm 0.82$ & $3.98 \pm 0.86$ & $3.98 \pm 0.77$ & & \\
\hline
\end{tabular}

How available to you is the information that you need in your day-to-day life? ${ }^{4}$

\begin{tabular}{|c|c|c|c|c|c|c|}
\hline Women & $4.65 \pm 0.50$ & $4.64 \pm 0.51$ & $4.68 \pm 0.47$ & $4.63 \pm 0.57$ & \multirow[t]{2}{*}{0.242} & \multirow[t]{2}{*}{0.010} \\
\hline Men & $4.71 \pm 0.45$ & $4.55 \pm 0.51$ & $4.78 \pm 0.42$ & $4.77 \pm 0.43$ & & \\
\hline \multicolumn{7}{|c|}{ o what extent do you have the opportunity for leisure activities? ${ }^{4}$} \\
\hline Women & $4.33 \pm 0.70$ & $4.21 \pm 0.76$ & $4.51 \pm 0.57$ & $4.30 \pm 0.72$ & \multirow[t]{2}{*}{0.481} & 0.005 \\
\hline Men & $4.28 \pm 0.68$ & $4.00 \pm 0.66$ & $4.41 \pm 0.69$ & $4.35 \pm 0.65$ & & \\
\hline
\end{tabular}


Table 3 Mean Likert-Scores of the WHQOLBREF-Items Displayed by Race Distance (Continued)

\begin{tabular}{|c|c|c|c|c|c|c|}
\hline \multirow{2}{*}{\multicolumn{7}{|c|}{ How well are you able to get around? ${ }^{1}$}} \\
\hline & & & & & & \\
\hline Women & $4.84 \pm 0.37$ & $4.76 \pm 0.43$ & $4.91 \pm 0.29$ & $4.93 \pm 0.27$ & \multirow[t]{2}{*}{0.394} & \multirow[t]{2}{*}{0.007} \\
\hline Men & $4.88 \pm 0.33$ & $4.85 \pm 0.36$ & $4.87 \pm 0.34$ & $4.91 \pm 0.29$ & & \\
\hline \multicolumn{7}{|c|}{ How satisfied are you with your sleep? ${ }^{2}$} \\
\hline Women & $3.82 \pm 0.91$ & $3.83 \pm 0.95$ & $3.81 \pm 0.85$ & $3.81 \pm 0.96$ & \multirow[t]{2}{*}{0.530} & \multirow[t]{2}{*}{0.005} \\
\hline Men & $3.96 \pm 0.85$ & $4.12 \pm 0.76$ & $4.00 \pm 0.81$ & $3.79 \pm 0.97$ & & \\
\hline \multicolumn{7}{|c|}{ How satisfied are you with your ability to perform your daily living activities? ${ }^{2}$} \\
\hline Women & $4.26 \pm 0.72$ & $4.23 \pm 0.75$ & $4.18 \pm 0.74$ & $4.52 \pm 0.58$ & \multirow[t]{2}{*}{0.226} & \multirow[t]{2}{*}{0.011} \\
\hline Men & $4.34 \pm 0.57$ & $4.21 \pm 0.49$ & $4.39 \pm 0.54$ & $4.40 \pm 0.66$ & & \\
\hline \multicolumn{7}{|c|}{ How satisfied are you with your capacity for work? ${ }^{2}$} \\
\hline Women & $4.23 \pm 0.71$ & $4.23 \pm 0.67$ & $4.18 \pm 0.69$ & $4.33 \pm 0.88$ & \multirow[t]{2}{*}{0.884} & \multirow[t]{2}{*}{0.001} \\
\hline Men & $4.46 \pm 0.61$ & $4.45 \pm 0.56$ & $4.43 \pm 0.62$ & $4.49 \pm 0.63$ & & \\
\hline \multicolumn{7}{|c|}{ How satisfied are you with yourself? ${ }^{2}$} \\
\hline Women & $3.96 \pm 0.72$ & $3.95 \pm 0.73$ & $3.91 \pm 0.66$ & $4.11 \pm 0.80$ & \multirow[t]{2}{*}{0.095} & \multirow[t]{2}{*}{0.017} \\
\hline Men & $4.14 \pm 0.61$ & $4.09 \pm 0.77$ & $4.28 \pm 0.46$ & $4.02 \pm 0.60$ & & \\
\hline \multicolumn{7}{|c|}{ How satisfied are you with your personal relationships? ${ }^{2}$} \\
\hline Women & $4.02 \pm 0.82$ & $4.05 \pm 0.80$ & $3.96 \pm 0.84$ & $4.04 \pm 0.85$ & \multirow[t]{2}{*}{0.699} & \multirow[t]{2}{*}{0.003} \\
\hline Men & $4.08 \pm 0.80$ & $4.00 \pm 0.71$ & $4.11 \pm 0.85$ & $4.12 \pm 0.82$ & & \\
\hline \multicolumn{7}{|c|}{ How satisfied are you with your sex life? ${ }^{2}$} \\
\hline Women & $3.58 \pm 0.96$ & $3.64 \pm 0.94$ & $3.51 \pm 1.04$ & $3.59 \pm 0.84$ & \multirow[t]{2}{*}{0.698} & \multirow[t]{2}{*}{0.003} \\
\hline Men & $3.58 \pm 1.09$ & $3.48 \pm 1.15$ & $3.54 \pm 1.13$ & $3.70 \pm 1.01$ & & \\
\hline \multicolumn{7}{|c|}{ How satisfied are you with the support you get from your friends? ${ }^{2}$} \\
\hline Women & $3.99 \pm 0.73$ & $3.87 \pm 0.68$ & $4.11 \pm 0.80$ & $4.07 \pm 0.68$ & \multirow[t]{2}{*}{0.423} & \multirow[t]{2}{*}{0.006} \\
\hline Men & $3.85 \pm 0.75$ & $3.76 \pm 0.61$ & $4.04 \pm 0.82$ & $3.72 \pm 0.73$ & & \\
\hline \multicolumn{7}{|c|}{ How satisfied are you with the conditions of your living place? ${ }^{2}$} \\
\hline Women & $4.13 \pm 0.90$ & $4.03 \pm 0.94$ & $4.21 \pm 0.90$ & $4.22 \pm 0.75$ & \multirow[t]{2}{*}{0.861} & \multirow[t]{2}{*}{0.001} \\
\hline Men & $4.14 \pm 0.91$ & $3.91 \pm 0.98$ & $4.20 \pm 0.96$ & $4.26 \pm 0.79$ & & \\
\hline How satisfie & with your acc & Ith services? ${ }^{2}$ & & & & \\
\hline Women & $4.19 \pm 0.81$ & $4.00 \pm 0.85$ & $4.39 \pm 0.75$ & $4.33 \pm 0.68$ & 0.403 & 0.007 \\
\hline Men & $4.25 \pm 0.75$ & $4.18 \pm 0.73$ & $4.33 \pm 0.70$ & $4.21 \pm 0.83$ & & \\
\hline How satisfie & with your trar & & & & & \\
\hline Women & $4.25 \pm 0.79$ & $4.24 \pm 0.69$ & $4.25 \pm 0.87$ & $4.30 \pm 0.87$ & 0.073 & 0.019 \\
\hline Men & $4.36 \pm 0.77$ & $4.03 \pm 0.85$ & $4.54 \pm 0.66$ & $4.42 \pm 0.76$ & & \\
\hline How often o & e negative fe & $\mathrm{h}$ as blue mood, & xiety, depress & & & \\
\hline Women & $3.80 \pm 0.80$ & $3.80 \pm 0.82$ & $3.65 \pm 0.79$ & $4.11 \pm 0.70$ & 0.109 & 0.016 \\
\hline Men & $4.37 \pm 0.65$ & $4.39 \pm 0.61$ & $4.37 \pm 0.68$ & $4.35 \pm 0.65$ & & \\
\hline
\end{tabular}

Note. Results are presented as mean \pm SD. 10 km - 10 Kilometer Control Group. HM - Half Marathon. M - Marathon. UM - Ultramarathon. p - p-value for ANOVA test. $\eta^{2}-$ effect size

${ }^{1} 1$ = very poor, $2=$ poor, $3=$ neither poor nor good, $4=$ good, $5=$ very good

${ }^{2} 1=$ very dissatisfied, $2=$ dissatisfied, $3=$ neither satisfied nor dissatisfied, $4=$ satisfied, $5=$ very satisfied

${ }^{3} 1=$ not at all, $2=$ a little, $3=$ a moderate amount, $4=$ very much, $5=$ an extreme amount

${ }^{4} 1=$ not at all, $2=$ a little, $3=$ moderately, $4=$ mostly, $5=$ completely

${ }^{5} 1$ = never, 2 = seldom, 3 = quite often, 4 = very often, $5=$ always

longer than men on average, researchers have found that women are more likely to report poorer health, suffer higher rates of morbidity, and use more health services than men $[43,44]$. In terms of social relationship scores, there were no detectable differences between men and women, which contradicts results of previous studies [17, 18]. This can be explained by the fact that athletes usually have higher scores in this domain and thus any sex 
difference was eliminated [45]. In environment scores, there were no sex differences either. This finding is consistent with the results from other research [14].

\section{Impact of the choice of the diet on quality of life}

A second important finding was that diet choice does not affect the QOL-domains of physical health, psychological wellbeing, and social relationships for women or environment for men. However, our subjects showed that mean total domain scores are constantly high level (i.e. 16.99 on the 4-20 scale), mainly exceeding scores that have been generated for the general population in other studies (i.e. 15.70 [46] and 15.22 [47] on the 4-20 scale).

These findings confirmed our hypothesis that QOL of runners who adhere to a vegetarian or vegan diet is as good as the QOL of those who follow an omnivorous diet. Thus, they supported the notion that a vegetarian or vegan diet can be an appropriate and an equivalent alternative to an omnivorous diet.

The results are consistent with current research. Several studies have shown high QOL scores in vegetarians [13] and vegans $[10,48]$. A reasonable explanation is the fact that a diet rich in fruit and vegetables leads to a higher degree of fitness and lower morbidity, and thus to a good health status $[5,7,49]$. It is beyond debate that a healthy body is an inevitable requirement for a healthy mind - and hence for a high perception of QOL [50]. The dictum 'Mens sana in corpore sano' - 'a healthy mind in a healthy body' - takes up this idea and also applies vice versa. This assumption has been supported by studies showing that vegetarians and vegans report low stress levels and good states of mood [21, 22].

Moreover, the high QOL scores can be explained by the personality profiles as well as moral concepts and personal beliefs of vegetarians and vegans. A current investigation shows that they tend to be more liberal, altruistic, universalistic, and empathic [48] and often deal intensively with moral and ethical concerns relating to animal treatment and conscious behavior towards the environment [49]. This could make them believe that they contribute to a sustainable relationship between mankind and environment [50], which could generate a higher life satisfaction.

However, we found a minor effect of diet on social relationships scores for men. This result can be explained again by men's self-perception or awareness of other men. The fact that men often still stick to traditional role concepts $[41,42]$ could lead them to consider male vegetarians or vegans as not being real men, since a real man has to eat meat [51]. This would evoke the impression of being isolated and excluded, consequently leading to a reduction in self-esteem and thus to lower life satisfaction. In addition, current literature reveals that vegetarians and vegans more often report that they neither live with a partner nor are married, respectively $[52,53]$. This tendency could be identified in our sample as well. Since it is well known that having a girlfriend/ boyfriend or wife/husband leads to a certain degree of life satisfaction [54] and, beyond that, prevents affective disorders such as depression [55], this fact could have caused lower scores as well.

Furthermore, our female subjects who adhered to an omnivorous diet had higher environment scores than the vegetarians/vegans. This finding was surprising because it was not consistent with existing literature. Since consumption of fruits and vegetables and thus vegetarianism/ veganism is regarded to be associated with a good socioeconomic background [56], we had expected that this would lead to high scores in financial resources, access to health and social care, and opportunities for acquiring new information and skills, which are the facets incorporated in the dimension environment. However, our subjects may have considered other facets in this dimension, for example, freedom, physical safety and physical environment, to be more important. As vegetarians and vegans usually have high demands concerning these topics, especially in the matter of physical environment $[23,57]$, this might have made them state lower satisfaction in this regard.

\section{Impact of the race distance on quality of life}

A third important finding was that our data did not show an interaction between race distance and physical health, psychological well-being and social relationships for women men.

In addition to the fact that mean QOL-scores of our subjects were consistently high, these results suggest that endurance running leads to a high degree of life satisfaction, regardless of the race distance. The findings are consistent with other research results $[33,58,59]$. There are various reasons which could explain this.

Similar to a well-balanced diet, physical activity in general, and endurance running in particular, are crucial factors which affect health. In this context, the 'healthy mind in a healthy body'-concept, which has already been mentioned before, could again provide an explanation [60,61]. Research into endocrine responses to exercise has shown a positive correlation between endurance training and endorphin levels [62]. Since endorphins are regarded to be responsible for good mood and a reduction in sensation of pain [63], these changes lead to a lower level of perceived stress and thus to well-being. Similar tendencies can be found for stress and anxiety perception in athletes. Endurance running in particular leads to a higher resilience to stress and anxiety [64], a good sleep architecture [30], and an increased self-perception specifically in terms of a perceived internal and body competence [65]. As both the NURMI-Runners and the members of the $10-\mathrm{km}$ 
control group derived high scores in the physical and psychological well-being dimensions, it appears likely that the previous explanation applies to both groups.

Besides health, sleep and body consciousness, motivational concerns and personality profiles of endurance runners are the basis for their high life satisfaction. Most athletes run voluntarily and therefore they are motivated by intrinsic reasons, such as self-esteem, self-discovery, improved fitness, life meaning or personal goal achievement and challenge [66]. Since endurance running challenges both body and mind to an extreme degree [67, 68], finishing a marathon shows that someone can achieve her/his goals and knows or even expands her/his personal limitations or abilities. In this context, the ability of 'self-conquest' is a crucial factor that contributes to the perception of extraordinary and wonderful feelings, leading to a certain degree of happiness and hence high QOL scores [12]. Furthermore, several authors have investigated the personality profiles of endurance athletes. They were described as task-oriented rather than ego-oriented, health and financially conscious [69], extroverted [70] and self-sufficient [71]. Moreover, they would have a certain degree of emotional intelligence [72]. These character traits are typically regarded to be positive and thus have positive effects on social relationships - one dimension of the QOL-domains. Since there were no detectable differences between the NURMI-Runners and the members of the 10-km control group in this regard, our findings suggest that these character traits apply to endurance runners of any distance and are not limited to one subgroup.

Furthermore, our data demonstrated a minor effect of the race distance on environment scores for women, where half-marathoners had higher counts than the members of the $10-\mathrm{km}$ control group. Considering that the domain of environment was assessed using, among others, the categories financial resources, freedom and security, home environment, participation in leisure activities, and transport, the finding could be explained by the socioeconomic background of the related athletes. It has been reported that marathon runners tend to have an above average high socioeconomic status [2, 73]. Belonging to a high social class means having more financial resources, a better home environment and better access to transport.

Summarizing the effects of diet choice and race distance on QOL, it can be concluded that the dual approach of regular physical activity, i.e. endurance running, and conscious nutrition, i.e. a vegetarian/vegan diet, is a crucial factor in the derivation of the high QOL scores that were found in the subjects. Beyond that, these two factors are synergistic and thus mutually reinforcing [23], which increases their impact. Obviously, the positive effects of endurance running doesn't seem to depend on the race distance, as both of the NURMI-Runners and the $10-\mathrm{km}$ controls showed high QOL scores. Further research is warranted to determine the optimal balance within the dual approach of physical activity such as endurance running linked to vegetarian or vegan nutrition, in order to achieve cumulative effects [23] for a high QOL.

\section{Diet $\times$ race-distance-interaction and its impact on quality of life}

A fourth important finding was that our data did not reveal a diet $\times$ race distance interaction concerning physical health, psychological wellbeing or social relationships for women or men.

Diet choice immediately before running or the composition of the personal diet might be influenced by the announced race distance [74, 75]. However, there is no evidence that the choice of diet in general has an effect on the preferred race distance and vice versa. Thus, an interference of one of the variables with the other affecting the influence on QoL would have been unexpected.

Nevertheless, a moderate diet $\times$ race distance interaction on the environment score was shown for men, although no interaction was found for women. This result could again be explained by the socioeconomic background of the runners. As has already been mentioned above, marathon runners tend to have above average levels of intelligence quotient (IQ) and a high socioeconomic status $[2,73]$. High IQ scores $[76,77]$ and belonging to a high socioeconomic group is positively correlated with the ability to reflect critically about diet choice $[78,79]$. In this way, an interaction between diet choice and race distance is possible.

\section{Limitations and implications for future research}

Some limitations of our study should be noted. The survey is based on self-report, meaning that the reliability of the data depends on the conscientiousness of our subjects. However, we minimized this effect by using questions to control for diet and race distance.

Moreover, the small sample size and the pre-selection of our subjects, due to the fact that only highly motivated runners took part, led to a lack of statistical representativeness, which might have affected our results. Nonetheless, the high intrinsic motivation of the participants would have led to an increase in the accuracy of their answers and hence to a higher quality of the generated data.

\section{Practical applications}

Since our survey is the first to investigate QOL in endurance runners adhering to a vegetarian or vegan diet, the results might be important for researchers involved in implementing individualized dietary strategies for athletes and thus may be used as reference for future 
studies. Moreover, our data may support recreational and professional runners as well as their coaches in finding an optimized nutrition strategy. Not only athletes but also non-runners and physicians might get a better insight into appropriate diets and more active lifestyles, and thus have a better basis for their choices for themselves, their families and even their patients. Beyond that, in the light of the aforementioned dual approach of regular physical activity integrated with vegetarian/vegan nutrition providing cumulative benefits for a high level of life satisfaction, the results might be used as a basis for public health and prevention programs for both children and adults.

\section{Conclusion}

In summary, our results reveal that the participants of our study, including the members of the $10-\mathrm{km}$ control group as well as the NURMI-Runners, had a high QOL, regardless of the race distance or diet choice. These findings contribute to a broad body of evidence supporting the notion that adhering to a vegetarian or vegan diet can be an appropriate and equal alternative to an omnivorous diet. In combination with an active lifestyle, i.e. by performing regular endurance running, this dual approach can be one way to effectively and successfully achieve a high degree of life satisfaction.

\section{Abbreviations}

10 km: 10-Kilometer Control Group; DOM: Domain; EKSG: Ethics Board of St. Gallen, Switzerland; HM: Half Marathon; M: Marathon; NURMI : Nutrition and Running High Mileage; QOL: Quality of Life; SD: Standard Deviation; UM: Ultramarathon; WHOQOLBREF: World Health Organization Quality of Life Assessment - brief version (french: bref)

\section{Acknowledgements}

The authors are grateful to the WHO, Health Statistics and Health Information Systems (HSI), Geneva Switzerland, for the permission (November 25, 2014) to use the English and German versions of the WHOQOL-BREF form in order to study the QOL within the NURMI Study (Step 2).

\section{Availability of data and materials}

The datasets generated and/or analyzed during the current study are not publicly available due to data security reasons but may be made available and provided by the principal investigator upon reasonable request. Subjects will receive a brief summary of the results of the NURMI Study if desired.

\section{Authors' contributions}

KW conceptualized, designed and developed the study design and the questionnaires together with BK and CL. PN performed data analysis. PB drafted the manuscript, TR helped in drafting the manuscript, and BK and KW critically reviewed it. Technical support was provided by GW and ChL. All authors read and approved the final manuscript.

\section{Ethics approval and consent to participate}

The study protocol is available online via https://ink.springer.com/article/ 10.1186/s40064-016-2126-4 and was approved by the ethics board of St. Gallen, Switzerland on May 6, 2015 (EKSG 14/145).

The study is conducted in accordance with the ethical standards of the institutional review board, medical professional codex and the with the 1964 Helsinki declaration and its later amendments as of 1996 as well as Data Security Laws and good clinical practice guidelines.

Study participation is voluntary and can be cancelled at any time without provision of reasons and without negative consequences.
Informed consent was obtained from all individual participants included in the study considering the data collected, used and analyzed exclusively and only in the context of the NURMI Study.

\section{Consent for publication}

Not applicable.

\section{Competing interests}

The authors declare that they have no competing interests.

\section{Publisher's Note}

Springer Nature remains neutral with regard to jurisdictional claims in published maps and institutional affiliations.

\section{Author details}

${ }^{1}$ Faculty of Medicine, University of Gießen, Gießen, Germany. ${ }^{2}$ Institute of Primary Care, University of Zurich, Zurich, Switzerland. ${ }^{3}$ Exercise Physiology Laboratory, Nikaia, Greece. ${ }^{4}$ ITEG, Innsbruck, Austria. ${ }^{5}$ AdventureV \& change2V, Stans, Austria. Institute of Nutrition, University of Gießen, Gießen, Germany. ${ }^{7}$ Institute of Primary Care, University of Zurich, Zurich, Switzerland. ${ }^{8}$ Centre for Research and Knowledge Management, Pedagogical University Tyrol, Feldstraße 1/II, 6010 Innsbruck, Austria. ${ }^{9}$ Department of Sport Science, University of Innsbruck, Innsbruck, Austria.

Received: 12 March 2018 Accepted: 26 June 2018

Published online: 17 July 2018

\section{References}

1. Running USA. Statistics. https://www.runningusa.org/research (2017). Accessed 08 Dec 2017.

2. Sport England. Active Lives Survey 2015-16. https://www.sportengland.org/ research/active-lives-survey/ (2016). Accessed 10 Nov 2017.

3. Wirnitzer KC, Leitzmann C, Knechtle B, Nikolaidis P, Wirnitzer G, Lechleitner C, Seyfart T, Boldt P. The NURMI study: methodology and first results of the prevalence of vegetarians and vegans in running events. Forsch Komplementmed. 2016;23(1):1-13.

4. Wirnitzer K, Seyfart T, Leitzmann C, Keller M, Wirnitzer G, Lechleitner C, Rüst $C$, Rosemann T, Knechtle B. Prevalence in running events and running performance of endurance runners following a vegetarian or vegan diet compared to non-vegetarian endurance runners: the NURMI study. Springerplus. 2016; https://doi.org/10.1186/s40064-016-2126-4.

5. Melina V, Craig W, Levin S. Position of the academy of nutrition and dietetics: vegetarian diets. J Acad Nutr Diet. 2016; https://doi.org/10.1016/j. jand.2016.09.025.

6. Rodriguez NR, Di Marco Langley NM. American College of Sports Medicine position stand. Nutrition and athletic performance. S Med Sci Sports Exerc. 2009; https://doi.org/10.1249/MSS.0b013e31890eb86.

7. Williams PT. Interactive effects of exercise, alcohol, and vegetarian diet on coronary artery disease risk factors in 9242 runners: the National Runners' health study. Am J Clin Nutr. 1997;66:1197-206.

8. WHO. WHOQOL-BREF: Introduction, Administration and Scoring. Field Trial version 1996. http://apps.who.int/iris/handle/10665/63529 (1996). Accessed 20 May 2017.

9. Baumann C, Erpelding ML, Régat S, Collin JF, Briançon S. The WHOQOLBREF questionnaire: French adult population norms for the physical health, psychological health and social relationship dimensions. Rev Epidemiol Sante Publique. 2010; https://doi.org/10.1016/.jespe.2009.10.009.

10. Agarwal U, Mishra S, Xu J, Levin S, Gonzales J, Barnard ND. A multicenter randomized controlled trial of a nutrition intervention program in a multiethnic adult population in the corporate setting reduces depression and anxiety and improves quality of life: the GEICO study. Am J Health Promot. 2015;29:245-54.

11. Gallicchio L, Hoffman SC, Helzlsouer KJ. The relationship between gender, social support, and health-related quality of life in a community-based study in Washington County Maryland. Qual Life Res. 2007;16:777-86.

12. Gill DL, Hammond CC, Reifsteck EJ, Jehu CM, Williams RA, Adams MM Lange EH, Becofsky K, Rodriguez E, Shang YT. Physical activity and quality of life. J Prev Med Public Health. 2013;46(1):28-34.

13. Kahleova H, Hrachovinova T, Hill M, Pelikanova T. Vegetarian diet in type 2 diabetes-improvement in quality of life, mood and eating behaviour. Diabet Med. 2013; https://doi.org/10.1111/dme.12032 . 
14. Kirchengast S, Haslinger B. Gender differences in health-related quality of life among healthy aged and old-aged Austrians: cross-sectional analysis. Gend Med. 2008; https://doi.org/10.1016/j.genm.2008.07.001.

15. Koikawa N, Shimada S, Suda S, et al. Sex differences in subjective sleep quality, sleepiness, and health-related quality of life among collegiate soccer players. Sleep Biol Rhythms. 2016; https://doi.org/10.1007/s41105-016-0068-4

16. Gholami A, Jahromi LM, Zarei E, Dehghan A. Application of WHOQOL-BREF in measuring quality of life in health-care staff. Int J Prev Med. 2013;4:809-17.

17. Zhang Y, Qu B, Lun S, Wang D, Guo Y, Liu J. Quality of life of medical students in China: a study using the WHOQOL-BREF. PLoS One. 2012; https://doi.org/10.1371/journal.pone.0049714.

18. Naumann VJ, Byrne GJ. WHOQOL-BREF as a measure of quality of life in older patients with depression. Int Psychogeriatr. 2004;16:159-73.

19. Liu X, Yan Y, Li F, Zhang D. Fruit and vegetable consumption and the risk of depression: a meta-analysis. Nutrition. 2016;32:296-302.

20. Opie RS, O'Neil A, Itsiopoulos C, Jacka FN. The impact of whole-of-diet interventions on depression and anxiety: a systematic review of randomised controlled trials. Public Health Nutr. 2015;18:2074-93.

21. Beezhold BL, Johnston CS, Daigle DR. Vegetarian diets are associated with healthy mood states: a cross-sectional study in seventh day Adventist adults. Nutr J. 2010; https://doi.org/10.1186/1475-2891-9-26

22. Beezhold B, Radnitz C, Rinne A, DiMatteo J. Vegans report less stress and anxiety than omnivores. Nutr Neurosci. 2015; https://doi.org/10.1179/ 1476830514Y.0000000164.

23. Wirnitzer KC. Vegan nutrition: latest boom in health and exercise. In AM Grumezescu \& AM Holban, editors. Therapeutic, Probiotic, and Unconventional Foods. Section 3: Unconventional Foods and Food Ingredients. Chapter 21. Academic Press, Elsevier; 2018. p. 387-453. ISBN: 978-0-12814-625-5. Available from URL: https://www.elsevier.com/books/therapeutic-probiotic-andunconventional-foods/grumezescu/978-0-12-814625-5. Accessed 25 Apr 2018.

24. Allen MW, Wilson M, Ng SH, Dunne M. Values and beliefs of vegetarians and omnivores. J Soc Psychol. 2000;140:405-22.

25. Houston MN, Hoch MC, Hoch JM. Health-related quality of life in athletes: a systematic review with Meta-analysis. J Athl Train. 2016; https://doi.org/10. 4085/1062-6050-51.7.03.

26. Beniamini Y, Rubenstein JJ, Zaichkowsky LD, Crim MC. Effects of highintensity strength training on quality-of-life parameters in cardiac rehabilitation patients. Am J Cardiol. 1997;80:841-6.

27. Crane M, Rissel C, Standen C, Greaves S. Associations between the frequency of cycling and domains of quality of life. Health Promot J Austr. 2014; https://doi.org/10.1071/HE14053.

28. Kell RT, Bell G, Quinney A. Musculoskeletal fitness, health outcomes and quality of life. Sports Med. 2001;31:863-73.

29. Maher JP, Pincus AL, Ram N, Conroy DE. Daily physical activity and life satisfaction across adulthood. Dev Psychol. 2015;51:1407-19.

30. Kredlow MA, Capozzoli MC, Hearon BA, Calkins AW, Otto MW. The effects of physical activity on sleep: a meta-analytic review. J Behav Med. 2015;38:427-49.

31. Gerber M, Pühse U. Do exercise and fitness protect against stress-induced health complaints? A review of the literature. Scand J Public Health. 2009;37:801-19.

32. Wegner M, Helmich I, Machado S, Nardi AE, Arias-Carrion O, Budde H. Effects of exercise on anxiety and depression disorders: review of meta- analyses and neurobiological mechanisms. CNS Neurol Disord Drug Targets. 2014;13:1002-14.

33. Knechtle B, Quarella A. Running helps-or how you escape depression without a psychiatrist and end up running a marathon. Praxis (Bern 1994). 2007:96:1351-6.

34. WHO. WHOQOL-BREF: Introduction, Administration and Scoring. Field Trial version 1996. http://apps.who.int/iris/handle/10665/63529. 1996. Accessed 20 May 2017.

35. Nørholm V, Bech P. The WHO quality of life (WHOQOL) questionnaire: Danish validation study. Nord J Psychiatry. 2001;55:229-35.

36. WHOQOL Group. Development of the World Health Organization WHOQOL-BREF quality of life assessment. Psychol Med. 1998;28:551-8.

37. Backovic DV, Zivojinovic Jl, Maksimovic J, Maksimovic M. Gender differences in academic stress and burnout among medical students in final years of education. Psychiatr Danub. 2012;24:175-81.

38. Moffat KJ, McConnachie A, Ross S, Morrison JM. First year medical student stress and coping in a problem-based learning medical curriculum. Med Educ. 2004;38:482-91.

39. Schaal K, Tafflet M, Nassif H, et al. Psychological balance in high level athletes: gender-based differences and sport-specific patterns. PLoS One. 2011; https://doi.org/10.1371/journal.pone.0019007
40. Kroenke K, Spitzer RL. Gender differences in the reporting of physical and somatoform symptoms. Psychosom Med. 1998;60:150-5.

41. Brannon RC. No 'Sissy stuff': the stigma of anything vaguely feminine. In: David D, Brannon RC, editors. The forty-nine percent majority. Reading: MA: Addison-Wesley; 1976. p. 49-50.

42. Sieverding M. Gender and health-related attitudes: The role of a 'Macho' Self/Concept. In: Weidner, et al., editors. Heart disease: environment, stress and gender. Amsterdam: IOS Press; 2002. p. 237-50.

43. Liu H. Gender paradox (and the health myth). In: The Wiley Blackwell encyclopedia of health, illness, behavior, and society; 2014. https://doi.org/ 10.1002/9781118410868.wbehibs 110 .

44. Revenson TA, Marín-Chollom AM. Gender differences in physical health. The Encyclopedia of Adulthood and Aging. 2015; https://doi.org/10.1002/ 9781118521373.wbeaa053.

45. Pucci G, Reis RS, Rech CR, Hallal PC. Quality of life and physical activity among adults: population-based study in Brazilian adults. Qual Life Res. 2012:21:1537-43.

46. Omorou YA, Erpelding ML, Escalon H, Vuillemin A. Contribution of taking part in sport to the association between physical activity and quality of life. Qual Life Res. 2013; https://doi.org/10.1007/s11136-013-0355-3.

47. Correia RF, Ribeiro AN, Barbieri JF, Brasil D, Motta L, Castaño LAA, Salve MGC. Quality of life levels in Brazilian elite female college volleyball players. Int J Sport Sci. 2017;7(1):6-9.

48. Kessler CS, Holler S, Joy S, Dhruva A, Michalsen A, Dobos G, Cramer H. Personality profiles, values and empathy: differences between lacto-Ovovegetarians and vegans. Forsch Komplementmed. 2016; https://doi.org/10. $1159 / 000445369$.

49. Ruby MB. Vegetarianism. A blossoming field of study. Appetite. 2012;58:141-50

50. Fox N, Ward KJ. Health, ethics and environment: a qualitative study of vegetarian motivations. Appetite. 2008;50:422-9.

51. Schösler $\mathrm{H}$, de Boer J, Boersema JJ, Aiking H. Meat and masculinity among young Chinese, Turkish and Dutch adults in the Netherlands. Appetite. 2015; https://doi.org/10.1016/j.appet.2015.02.013.

52. Baines S, Powers J, Brown W. How does the health and well-being of young Australian vegetarian and semi-vegetarian women compare with nonvegetarians? Public Health Nutr. 2007; https://doi.org/10.1017/ S1368980007217938

53. Michalak J, Zhang XC, Jacobi F. Vegetarian diet and mental disorders: results from a representative community survey. Int J Behav Nutr Phys Act. 2012; https://doi.org/10.1186/1479-5868-9-67.

54. Han KT, Park EC, Kim JH, Kim SJ, Park S. Is marital status associated with quality of life? Health Qual Life Outcomes. 2014; https://doi.org/10.1186/ s12955-014-0109-0.

55. Scott KM, Wells JE, Angermeyer M, et al. Gender and the relationship between marital status and first onset of mood, anxiety and substance use disorders. Psychol Med. 2010; https://doi.org/10.1017/S0033291709991942.

56. Estaquio C, Druesne-Pecollo N, Latino-Martel P, Dauchet L, Hercberg S, Bertrais $\mathrm{S}$. Socioeconomic differences in fruit and vegetable consumption among middle-aged French adults: adherence to the 5 a day recommendation. J Am Diet Assoc. 2008;108:2021-30.

57. Lindeman M, Sirelius M. Food choice ideologies: the modern manifestations of normative and humanist views of the world. Appetite. 2001;37:175-84.

58. Bäckmand H, Kaprio J, Kujala U, Sarna S. Personality and mood of former elite male athletes - a descriptive study. Int J Sports Med. 2001; https://doi. org/10.1055/s-2001-16382.

59. Sato M, Jordan JS, Funk DC. Distance running events and life satisfaction: a longitudinal study. J Sport Manage. 2015;29:347-61.

60. Bize R, Johnson JA, Plotnikoff RC. Physical activity level and health-related quality of life in the general adult population: a systematic review. Prev Med. 2007:45:401-15.

61. Brown DW, Balluz LS, Heath GW, Moriarty DG, Ford ES, Giles WH, Mokdad $\mathrm{AH}$. Associations between recommended levels of physical activity and health-related quality of life. Findings from the 2001 behavioral risk factor surveillance system (BRFSS) survey. Prev Med. 2003;37:520-8.

62. Knechtle B. Influence of physical activity on mental well-being and psychiatric disorders. Praxis (Bern 1994). 2004;93:1403-11.

63. Boecker H, Sprenger T, Spilker ME, Henriksen G, Koppenhoefer M, Wagner KJ, Valet M, Berthele A, Tolle TR. The runner's high: opioidergic mechanisms in the human brain. Cereb Cortex. 2008;18:2523-31.

64. Salmon P. Effects of physical exercise on anxiety, depression, and sensitivity to stress a unifying theory. Clin Psychol Rev. 2001;21:33-61. 
65. Skrinar GS, Bullen BA, Cheek JM, MCArthur JW, Vaughan LK. Effects of endurance training on body-consciousness in women. Percept Mot Skills. 1986;62:483-90.

66. Boudreau AL, Giorgi B. The experience of self-discovery and mental change in female novice athletes in connection to marathon running. J Phenomenol Psychol. 2010;41:234-67.

67. Cona G, Cavazzana A, Paoli A, Marcolin G, Grainer A, Bisiacchi PS. It's a matter of mind! Cognitive functioning predicts the athletic performance in ultra-Marathon runners. PLoS One. 2015; https://doi.org/10.1371/journal. pone.0132943

68. Sjödin B, Svedenhag J. Applied physiology of marathon running. Sports Med. 1985;2:83-99.

69. Krouse RZ, Ransdell LB, Lucas SM, Pritchard ME. Motivation, goal orientation coaching, and training habits of women ultrarunners. J Strength Cond Res. 2011; https://doi.org/10.1519/JSC.0b013e318204caa0.

70. Egloff B, Gruhn AJ. Personality and endurance sports. Pers Individ Dif. 1996; 21:223-9.

71. Hartung GH, Farge EJ. Personality and physiological traits in middle-aged runners and joggers. J Gerontol. 1977;32:541-8.

72. Lane AM, Wilson M. Emotions and trait emotional intelligence among ultraendurance runners. J Sci Med Sport. 2011; https://doi.org/10.1016/j.jsams. 2011.03.001

73. Valentine AS. The middle-aged marathon runner. Can Fam Physician. 1982; 28:941-5.

74. Arciero PJ, Miller VJ, Ward E. Performance enhancing diets and the PRISE protocol to optimize athletic performance. J Nutr Metab. 2015; https://doi. org/10.1155/2015/715859.

75. Williamson E. Nutritional implications for ultra-endurance walking and running event. Extrem Physiol Med. 2016; https://doi.org/10.1186/s13728016-0054-0.

76. Gale CR, Deary IJ, Schoon I, Batty GD. IQ in childhood and vegetarianism in adulthood: 1970 British cohort study. BMJ. 2007;334:245.

77. Moreira PA, Padrão PD. Educational and economic determinants of food intake in Portuguese adults: a cross-sectional survey. BMC Public Health. 2004; https://doi.org/10.1186/1471-2458-4-58.

78. Pecheya R, Monsivaisb P. Socioeconomic inequalities in the healthiness of food choices: exploring the contributions of food expenditures. Prev Med. 2016; https://doi.org/10.1016/j.ypmed.2016.04.012.

79. Pollard J, Greenwood D, Kirk S, Cade J. Lifestyle factors affecting fruit and vegetable consumption in the UK Women's cohort study. Appetite. 2001;37:71-9.

Ready to submit your research? Choose BMC and benefit from:

- fast, convenient online submission

- thorough peer review by experienced researchers in your field

- rapid publication on acceptance

- support for research data, including large and complex data types

- gold Open Access which fosters wider collaboration and increased citations

- maximum visibility for your research: over $100 \mathrm{M}$ website views per year

At BMC, research is always in progress.

Learn more biomedcentral.com/submissions 